\title{
Enhanced recovery implementation in colorectal surgery_-temporary or persistent improvement?
}

\author{
David Martin ${ }^{1}$ • Didier Roulin ${ }^{1}$ • Valérie Addor ${ }^{1}$. Catherine Blanc ${ }^{2}$. \\ Nicolas Demartines ${ }^{1} \cdot$ Martin Hübner $^{1}$
}

Received: 1 May 2016 / Accepted: 27 September 2016/Published online: 11 October 2016

(C) Springer-Verlag Berlin Heidelberg 2016

\begin{abstract}
Purpose Enhanced recovery after surgery (ERAS) implementation has proven to reduce complication rate and length of hospital stay. Little is known about the sustainability of these results over time. The study aim was to assess the application of ERAS pathway over the first 4 years after initial implementation.

Methods This retrospective study analyzed data collected prospectively from 482 consecutive elective colorectal patients operated in 2011 during the ERAS implementation process $(n=66)$, and after initial implementation in $2012(n=136)$, $2013(n=152)$, and $2014(n=128)$. Auditing ERAS was appraised from different perspectives: adherence with the ERAS protocol, clinical outcomes, and functional recovery. Patients were compared by year.

Results The groups were similar in terms of demographics and surgical strategies. Length of stay remained stable at 7 days without significant variation over time $(p=0.741)$. The 30-day complications and readmission rates were not statistically different (respectively $p=0.068$ and $p=0.639$ ). There was also no difference in functional recovery, more particularly, first flatus occurred at day $2(p=0.177)$, pain was adequately controlled with oral analgesics at day 2
\end{abstract}

Presented to the 102th Annual Congress of the Swiss Society of Surgery, 2015, Bern, Switzerland; published in abstract form as Swiss Knife 2015; 12: special edition

Nicolas Demartines

demartines@chuv.ch

1 Department of Visceral Surgery, University Hospital CHUV, Lausanne, Switzerland

2 Department of Anaesthesiology, University Hospital CHUV, Lausanne, Switzerland
( $p=0.111$ ), and patients were mobilized more than $4 \mathrm{~h}$ the first postoperative day in more than $60 \%$ of cases $(p=0.343)$. Overall adherence with the ERAS pathway was $73 \%$ during implementation and, respectively, 73 and $77 \%$ for 2012 and 2013 after initial implementation $(p=0.614)$. A significant decrease between $2013(77 \%)$ and $2014(73 \%)$ was observed $(p=0.032)$.

Conclusions Application of the institutional ERAS pathway could be maintained in the first 4 years after implementation leading to sustained improved functional recovery and clinical outcome.

Keywords Enhanced recovery $\cdot$ Implementation $\cdot$ Colorectal surgery $\cdot$ Sustainability

\section{Introduction}

Enhanced recovery after surgery (ERAS) pathways have proven to reduce overall morbidity, length of hospital stay, and costs $[1,2]$. Colorectal guidelines are updated regularly and currently include 21 care elements [3,4]. Adherence to these items is significantly associated with improved clinical outcomes [5, 6]. The implementation of such protocols, however, is a complex process and requires initially time and personal as well as financial investment [2]. Successful implementation has been practiced and reported in various countries and settings $[2,7,8]$. Unfortunately, very few data is available on sustainability of ERAS pathway over time [9]. Despite the benefits of the ERAS program, its implementation and adherence to the protocol remain a challenge and are rarely investigated.

The study aims were to assess the sustainability of the ERAS pathway for colorectal surgery after its initial implementation and to assess its influence on functional recovery 
and clinical outcomes over 4 years while considering adherence.

\section{Material and methods}

ERAS for colon and rectum surgery was implemented in our academic center in May 2011. The implementation period ended in November 2011 [2]. This retrospective study analyzed data collected prospectively from 482 consecutive colorectal patients operated in 2011 during the ERAS implementation process $(n=66)$ and after initial implementation in 2012 $(n=136), 2013(n=152)$, and until October $2014(n=128)$. Patients were compared by year. Of note, since April 2012, all emergent procedures are included in the ERAS program [10]. In this study, only elective procedure and those which were performed after unplanned admission with at least $24 \mathrm{~h}$ of hospitalization were included. The current analysis includes colorectal resections, while ileostomy and colostomy closures were excluded. As control group, 50 patients operated before ERAS implementation were assessed for ERAS adherence.

Auditing ERAS pathway was appraised according to different perspectives:

- Measuring adherence to the ERAS protocol. The measure of adherence has proven to be an instrumental tool to assess the success of ERAS pathway $[5,11]$.

- Measuring clinical outcomes of ERAS like length of stay, readmission rates, and complications according to the Clavien classification $[5,9,12]$.

- Determining functional recovery, defined as adequate pain control with oral analgesia (using a visual analog scale and pain quoted $<4 / 10$ ), recovery of intestinal motility (flatus), and mobilization more than $4 \mathrm{~h}$ the first postoperative day.

Prospective database documentation was performed by a dedicated and specially trained enhanced recovery nurse using ERAS Interactive Audit System. The Audit system analyzes the database for patients' characteristics, treatment, and outcomes. Moreover, all patients were examined by a surgeon at our center 4 to 6 weeks after discharge. The institutional review board approved the study, and all patients provided written consent before surgery.

Overall adherence to ERAS pathway was calculated as the number of elements fulfilled divided by 21 (total number of preoperative, intraoperative, and postoperative elements). Individual item adherence was calculated as percentage of compliant patients divided by the total of patients. Enemas were used for patients having a left-sided anastomosis and a stapler passing per rectal. These were counted as compliant with the ERAS pathway. Data on mobilization and oral fluid introduction was extracted from case notes of patients. Length of stay was counted from day of surgery to discharge. Descriptive statistics for categorical variables were reported as number and percentage, while continuous variables were reported as median and interquartile range or means and standard deviation as appropriate. Continuous variables were compared between periods with the Kruskal-Wallis test or Mann-Whitney $U$ test when appropriate. Pearson's chisquare test was used for comparison of categorical variables. A $p$ value $\leq 0.05$ was considered statistically significant. All statistical analyses were two-sided and performed using SPSS 22.0 software (SPSS Inc., Chicago, IL).

\section{Results}

Patients were similar in terms of demographics and performed surgical procedures (Table 1). Length of stay stabilized at a median of 7 days without significant difference between years $(p=0.741)$. Similarly, the 30-day complications and readmission rates were not statistically different (respectively $p=0.068$ and $p=0.639$, Table 2). Patients with an ASA score of 1 or 2 had a significantly lower complication rate than patients with a score of 3 or 4 (43.1 vs. $67.2 \%, p<0.001)$. There was no difference in functional recovery, more particularly, first flatus occurred at day $2(p=0.177)$, pain was adequately controlled by oral analgesics at day $2(p=0.111)$, and patients were mobilized more than $4 \mathrm{~h}$ the first postoperative day in more than $60 \%$ of cases $(p=0.343$, Table 3$)$.

ERAS items were applied in $41 \%$ of the 50 patients operated before implementation. This overall adherence increased significantly to $73 \%$ during implementation and then remained respectively at 73 and $77 \%$ for 2012 and 2013 after initial implementation $(p=0.614)$. A significant decrease between $2013(77 \%)$ and $2014(73 \%)$ was observed $(p=0.032$, Fig. 1). Adherences for each ERAS item according to the year are detailed in Figs. 2, 3, and 4. The adherence to postoperative items was lower than that to pre- and intraoperative items.

\section{Discussion}

Application of the institutional ERAS pathway could be maintained in the first 4 years after systematic implementation for colorectal surgery, despite decreased adherence during the last year. Consequently, improved functional recovery and clinical outcomes could be sustained.

A Dutch team implemented ERAS successfully in 33 hospitals and reported their follow-up at 3-5 years [7, 9]. Their overall trend showed stabilization in length of stay and functional recovery during the study period. However, protocol adherence decreased from 75 to $67 \%(p=0.32)$, which is comparable to our own results. Especially adherence to postoperative care elements dropped considerably. A Polish study 
Table 1 Patient demographics and performed surgical procedures during and after implementation of ERAS

\begin{tabular}{|c|c|c|c|c|c|}
\hline & \multirow{2}{*}{$\begin{array}{l}\text { During } \\
2011, n=66\end{array}$} & \multicolumn{3}{|l|}{ After } & \multirow[t]{2}{*}{$p$ value } \\
\hline & & $2012, n=136$ & $2013, n=152$ & $2014, n=128$ & \\
\hline Mean age (SD) & $64.9(18.2)$ & $61.9(17.5)$ & $64.8(15.1)$ & $63.2(15.6)$ & $0.523^{\mathrm{a}}$ \\
\hline Mean BMI in $\mathrm{kg} / \mathrm{m}^{2}$ (SD) & $25.3(4.7)$ & $25.1(4.5)$ & $26.2(4.5)$ & $26.1(5.9)$ & $0.194^{\mathrm{a}}$ \\
\hline Male/female & $36 / 30$ & $72 / 64$ & $79 / 73$ & $75 / 53$ & $0.709^{\mathrm{b}}$ \\
\hline Malignant/benign & $47 / 19$ & $83 / 53$ & $81 / 71$ & $73 / 55$ & $0.088^{\mathrm{b}}$ \\
\hline ASA score, $n(\%)$ & & & & & $0.451^{\mathrm{b}}$ \\
\hline $1-2$ & $52(78.8)$ & $100(73.5)$ & $104(68.4)$ & $92(71.9)$ & \\
\hline $3-4$ & $14(21.2)$ & $36(26.5)$ & $48(31.6)$ & $36(28.1)$ & \\
\hline Surgical procedure, $n(\%)$ & & & & & $0.129^{\mathrm{b}}$ \\
\hline Left colon & $31(47.0)$ & $54(39.7)$ & $75(49.3)$ & $58(45.3)$ & \\
\hline Right colon & $14(21.2)$ & $36(26.5)$ & $36(23.7)$ & $31(24.2)$ & \\
\hline (Sub)total colectomy & $1(1.5)$ & $7(5.1)$ & $13(8.6)$ & $3(2.3)$ & \\
\hline Rectum & $20(30.3)$ & $39(28.7)$ & $28(18.4)$ & $36(28.1)$ & \\
\hline Surgical approach, $n(\%)$ & & & & & $0.253^{\mathrm{b}}$ \\
\hline Laparoscopic & $46(69.7 \%)$ & $74(54.4)$ & $95(62.5)$ & $74(57.8)$ & \\
\hline Open & $14(21.2 \%)$ & $51(37.5)$ & $43(28.3)$ & $38(29.7)$ & \\
\hline Converted & $6(9.1 \%)$ & $11(8.1)$ & $14(9.2)$ & $16(12.5)$ & \\
\hline
\end{tabular}

ASA American Society of Anesthesiologists, $S D$ standard deviation

Left colon: left colectomy, sigmoid resection, Hartmann's reversal. Right colon: ileocecal and right colectomy. Rectum: (low) anterior resection, abdominoperineal resection, proctocolectomy

${ }^{\text {a }}$ Kruskal-Wallis test

${ }^{\mathrm{b}}$ Pearson's chi-square test showed that the introduction of the ERAS protocol is a gradual process, and its compliance at the level of $80 \%$ or more requires at least 30 patients and the period of about 6 months [13]. Several other studies have also shown that preoperative and intraoperative adherence remained almost equal over time, but postoperative adherence decreases generally $[9$,
14]. Postoperative elements were also the most difficult to implement [7]. Particularly, the cessation of intravenous fluids, early mobilization, and early resumption of solid foods did belong to old dogmas and was difficult to implement routinely. Randomized studies on the importance of the different ERAS components are lacking, and evaluating the impact of
Table 2 Clinical outcome during and after implementation of ERAS

\begin{tabular}{llllll}
\hline & During & After & & & $p$ value \\
\cline { 3 - 5 } & $2011, n=66$ & $2012, n=136$ & $2013, n=152$ & $2014, n=128$ & \\
\hline Median LOS (IQR) & $7(5-10)$ & $7(4-12)$ & $7(4-11)$ & $7(4-10)$ & $0.741^{\mathrm{c}}$ \\
30-day readmissions, (\%) & $1(1.5)$ & $7(5.1)$ & $8(5.3)$ & $6(4.7)$ & $0.639^{\mathrm{d}}$ \\
30-day complications ${ }^{\mathrm{a}, \mathrm{b}},(\%)$ & & & & & $0.068^{\mathrm{d}}$ \\
Overall & $32(48.5)$ & $80(58.8)$ & $69(45.4)$ & $59(46.1)$ & \\
Grades I-II & $26(39.4)$ & $60(44.1)$ & $45(29.6)$ & $40(31.3)$ & \\
Grades III-IV & $6(9.1)$ & $18(13.2)$ & $19(12.5)$ & $19(14.8)$ & \\
Mortality & $0(-)$ & $2(1.5)$ & $5(3.3)$ & $0(-)$ & \\
\hline
\end{tabular}

$L O S$ length of stay, IQR interquartile range

${ }^{\text {a }}$ According to the Clavien classification [12]

${ }^{\mathrm{b}}$ For comparison, the complication rates in 50 patients operated before implementation were respectively $36.2 \%$ for grades I-II, $17.0 \%$ for grades III-IV, and $55.3 \%$ for overall morbidity. The mortality rate was $2.1 \%$

${ }^{\mathrm{c}}$ Kruskal-Wallis test

${ }^{\mathrm{d}}$ Pearson's chi-square test 
Table 3 Functional recovery during and after implementation of ERAS

\begin{tabular}{llllll}
\hline & During & After & & $p$ value \\
\cline { 5 - 6 } & $2011, n=66$ & $2012, n=136$ & $2013, n=152$ & $2014, n=128$ \\
\hline POD when first passage of flatus (IQR) & $2(1-2)$ & $2(1-3)$ & $2(1-2)$ & $2(1-2)$ & $0.177^{\text {a }}$ \\
POD when oral pain control (IQR) & $2(1-4)$ & $2(1-3)$ & $2(1-3)$ & $2(1-3)$ & $0.111^{\text {a }}$ \\
Mobilization $>4$ h on POD1, $n(\%)$ & $44(66.7)$ & $87(64.0)$ & $110(72.4)$ & $81(63.3)$ & $0.343^{\text {b }}$ \\
\hline
\end{tabular}

$P O D$ postoperative day, $I Q R$ interquartile range

${ }^{a}$ Kruskal-Wallis test

${ }^{\mathrm{b}}$ Pearson's chi-square test

each single intervention is difficult since they probably influence each other, without evidence if every element is of equal importance [5]. One prospective observational study of more than 2000 colorectal cancer resections showed that specific elements from the program, namely laparoscopic surgery, restrictive intravenous fluids, preoperative carbohydrate and fluid loading, and intravenous anesthesia, were independently associated with improved outcome [6]. Another observational cross-sectional study showed that the only items clearly associated with reduced functional hospital stay were measures against hypothermia and mobilization before $24 \mathrm{~h}$ [15]. Although these studies indicate that certain elements are more important than others, it still remains to be elucidated if some may be omitted while not compromising outcomes.

A systematic audit of quality improvement is essential to maintain adherence to the clinical pathway and provide information for further improvements. Keeping adherence optimal remains an ongoing challenge that requires repeated training and dedicated personnel [16]. At the beginning of our experience in 2011, ERAS meetings were held twice a month until 2013, then once a month since 2014. Surgeons, anesthesiologists, and nursing staff take part in these regular meetings. Practical aspects and any necessary improvements are discussed to identify problems and find solutions. A subtle decrease in adherence was observed in 2014 compared to 2013. The difference cannot be definitively explained because the reasons for protocol deviations were not registered. As overall compliance in 2014 was similar as in 2011 and 2012, periodical fluctuations appear to be a plausible explication. Another potential explanation could be the decreased attention due to staff changes (residents and nurses) and less regular meetings. Bakker et al. showed that adherence to ERAS protocol was high in 2006 and 2007 (73\%), but decreased to $66 \%$ in 2008 and 2009, and up to $63 \%$ in 2010 and 2011
Fig. 1 Adherence with the ERAS pathway. * indicates statistical significance: $p$ value $\leq 0.05$, Mann-Whitney $U$ test

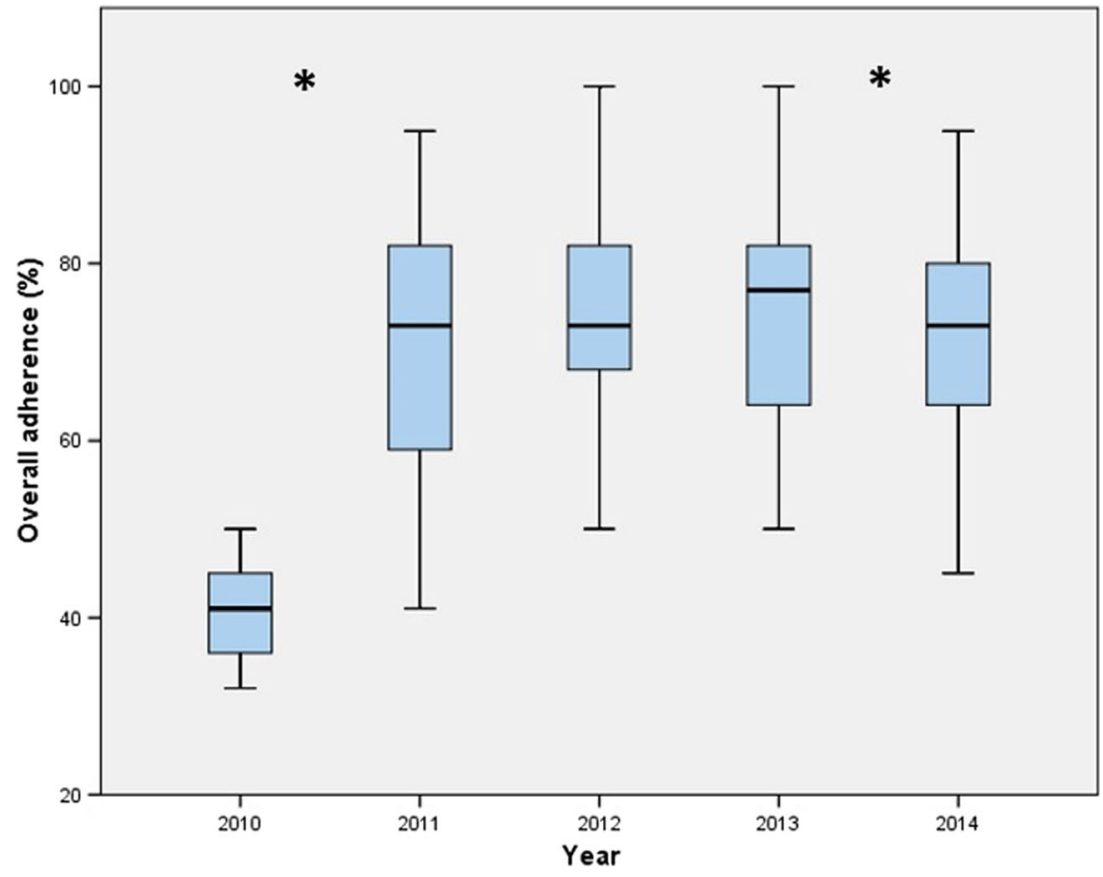


Fig. 2 Adherence to preoperative ERAS items (in percent). * indicates statistical significance: $p$ value $\leq 0.05$, Pearson's chi-square test

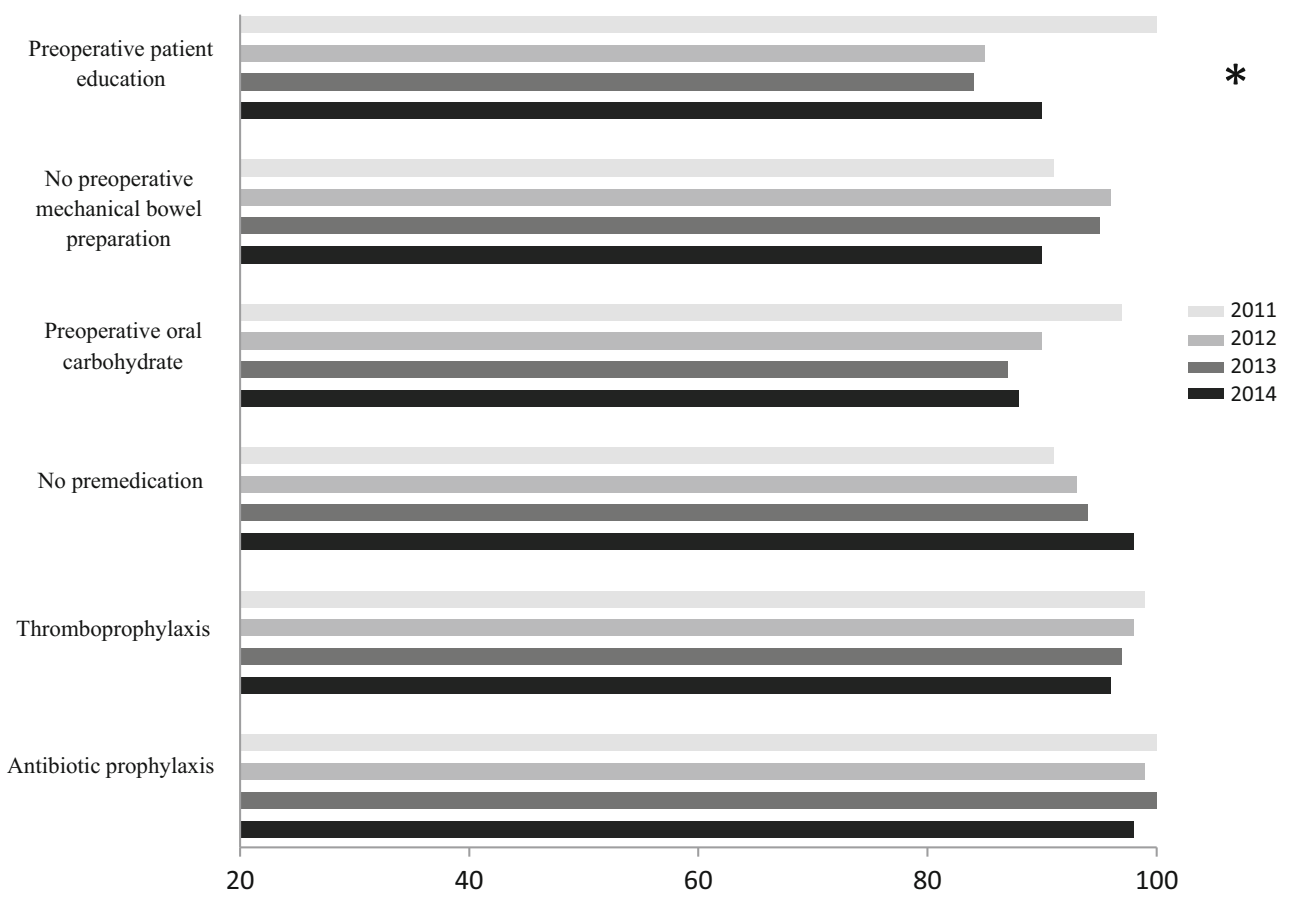

[16]. Only after specific measures at the outpatient clinic and the surgical ward, adherence improved again in 2012 and $2013(82 \%)$. Key factors include repeated training sessions and the presence of trained nurse-practitioners at all stages in perioperative care in order to prevent decreasing compliance after initial successful implementation [17].

An Australian study examined qualitatively the barriers to implementation of ERAS program [18]. This study based on interviews displayed four key areas, which include patientrelated factors, staff-related factors, practice-related factors, and finally health system resource aspects. In parallel with quantitative studies, qualitative studies are required as well to further define barriers to ERAS implementation. Markers to measure sustainability of such ERAS pathway are needed. Moreover, continuous re-evaluation of the evidences is mandatory, in order to update the guidelines [19].

This present study has several limitations besides its retrospective method and cannot be generalized to different countries and health systems. As shown by different studies, it may exist a confounding effect of postoperative recovery when assessing postoperative ERAS components as item compliance (cause) and not as early functional outcome (effect) as
Fig. 3 Adherence to intraoperative ERAS items (in percent). * indicates statistical significance: $p$ value $\leq 0.05$, Pearson's chi-square test. $P O N V$ postoperative nausea and vomiting

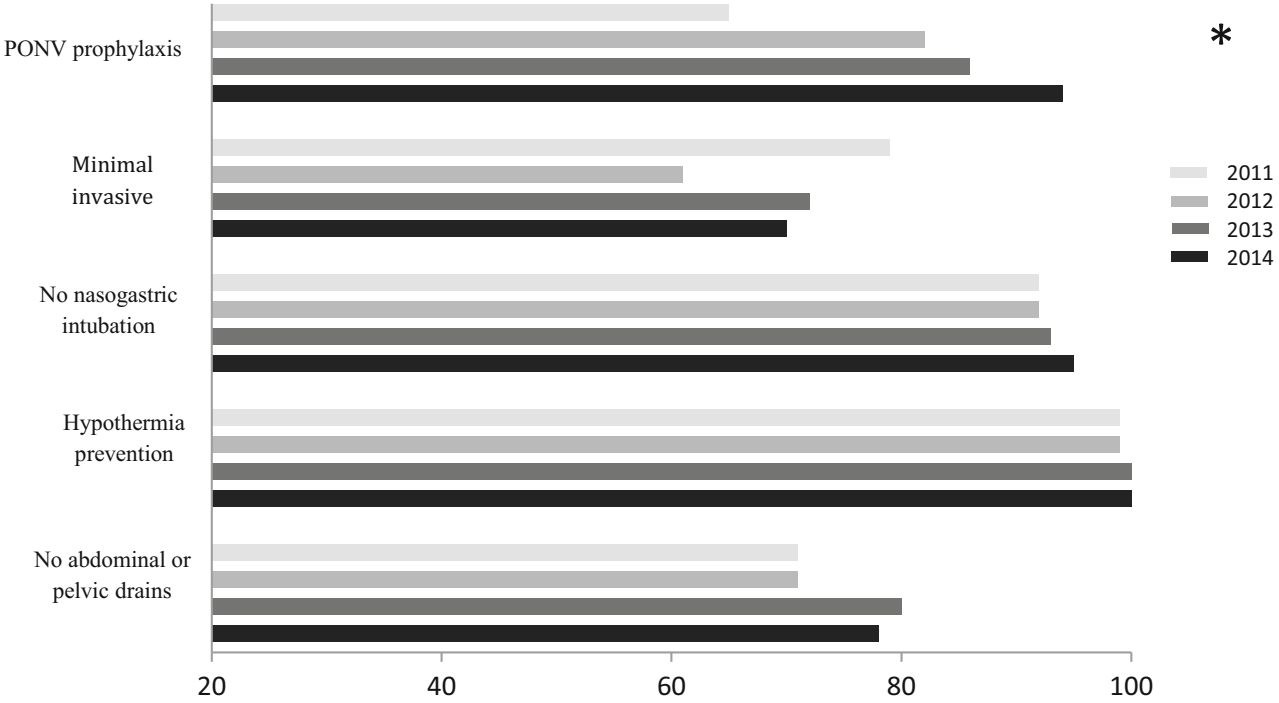


Fig. 4 Adherence to postoperative ERAS items (in percent). $*$ indicates statistical significance: $p$ value $\leq 0.05$, Pearson's chi-square test. $O D$ operative day, $P O D$ postoperative day

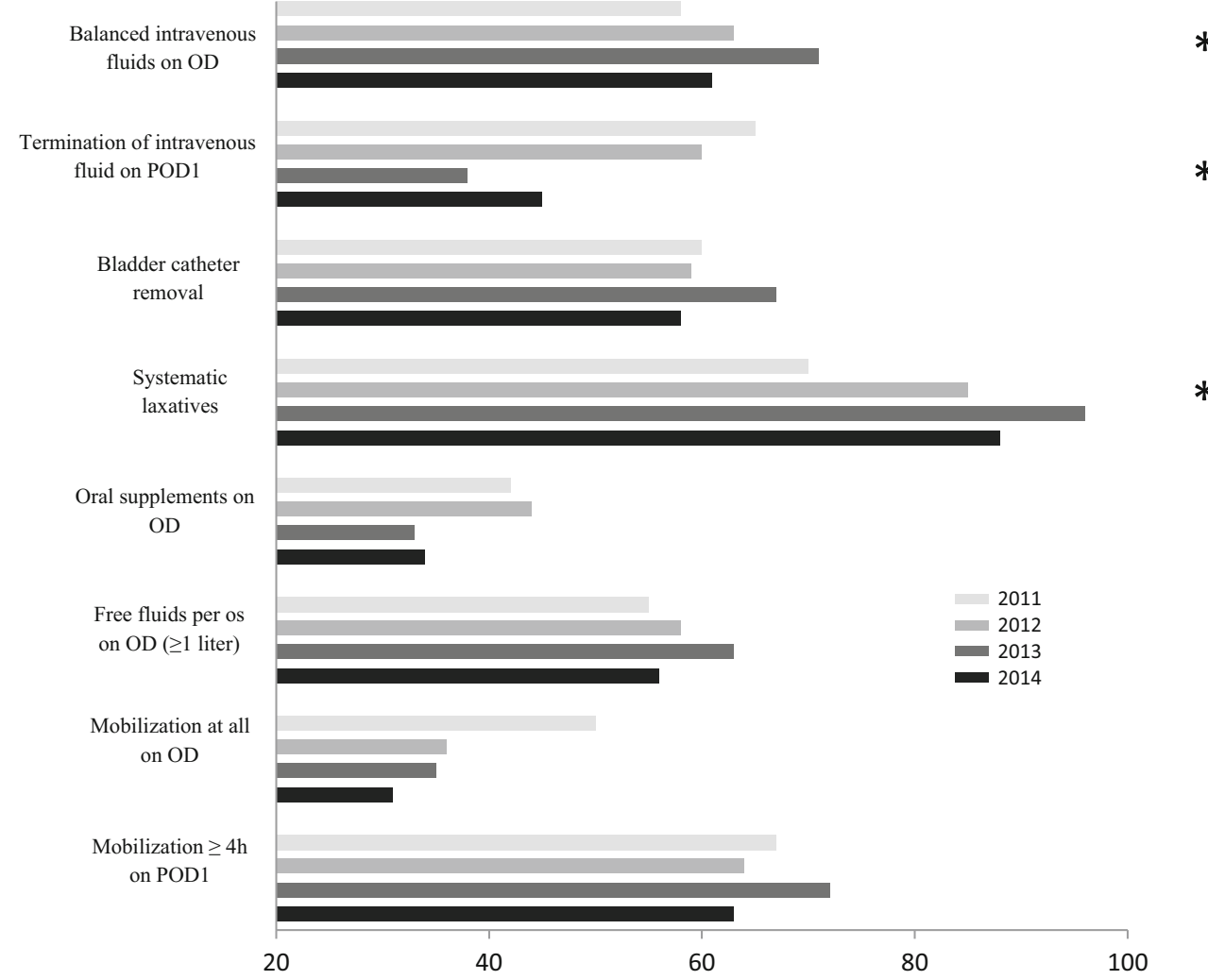

$*$

$*$ advocated by others $[20,21]$. All data was collected prospectively by data managers, but the present analysis was performed in retrospect; Hawthorne effect was thus prevented. A strength of the study is that implementation and sustainability were evaluated by combining three different criteria based on literature and on our own experience during a 4-year follow-up period.

\section{Conclusion}

Application of the institutional ERAS pathway could be maintained in the first 4 years after implementation leading to sustained improved functional recovery and clinical outcome.

Authors' contribution Study conception and design: DM, MH, and ND

Acquisition of data: DM and VA

Analysis and interpretation of data: DM, DR, CB, ND, and $\mathrm{MH}$

Drafting of manuscript: DM, DR, and MH

Critical revision of manuscript: DM, DR, VA, CB, ND, and MH

\section{Compliance with ethical standards}

Conflicts of interest The authors declare that they have no conflict of interest.

Ethical approval All procedures performed in studies involving human participants were in accordance with the ethical standards of the institutional and/or national research committee and with the 1964 Helsinki Declaration and its later amendments or comparable ethical standards.

Informed consent Informed consent was obtained from all individual participants included in the study.

Funding None.

\section{References}

1. Greco M, Capretti G, Beretta L, Gemma M, Pecorelli N, Braga M (2014) Enhanced recovery program in colorectal surgery: a metaanalysis of randomized controlled trials. World J Surg 38(6):15311541

2. Roulin D, Donadini A, Gander S, Griesser AC, Blanc C, Hubner M, Schafer M, Demartines N (2013) Cost-effectiveness of the implementation of an enhanced recovery protocol for colorectal surgery. Br J Surg 100(8):1108-1114

3. Gustafsson UO, Scott MJ, Schwenk W, Demartines N, Roulin D, Francis N, McNaught CE, Macfie J, Liberman AS, Soop M, Hill A, Kennedy RH, Lobo DN, Fearon K, Ljungqvist O (2013) Guidelines for perioperative care in elective colonic surgery: Enhanced Recovery After Surgery (ERAS®) Society recommendations. World J Surg 37(2):259-284

4. Nygren J, Thacker J, Carli F, Fearon KC, Norderval S, Lobo DN, Ljungqvist O, Soop M, Ramirez J (2013) Guidelines for perioperative care in elective rectal/pelvic surgery: Enhanced Recovery After Surgery (ERAS $®)$ Society recommendations. World J Surg 37(2):285-305 
5. Gustafsson UO, Hausel J, Thorell A, Ljungqvist O, Soop M, Nygren J (2011) Adherence to the enhanced recovery after surgery protocol and outcomes after colorectal cancer surgery. Arch Surg 146(5):571-577

6. The impact of enhanced recovery protocol compliance on elective colorectal cancer resection: results from an International Registry (2015). Ann Surg 261(6):1153-1159.

7. Gillissen F, Hoff C, Maessen JM, Winkens B, Teeuwen JH, von Meyenfeldt MF, Dejong CH (2013) Structured synchronous implementation of an enhanced recovery program in elective colonic surgery in 33 hospitals in the Netherlands. World J Surg 37(5): 1082-1093

8. Miller TE, Thacker JK, White WD, Mantyh C, Migaly J, Jin J, Roche AM, Eisenstein EL, Edwards R, Anstrom KJ, Moon RE, Gan TJ (2014) Reduced length of hospital stay in colorectal surgery after implementation of an enhanced recovery protocol. Anesth Analg 118(5):1052-1061

9. Gillissen F, Ament SM, Maessen JM, Dejong CH, Dirksen CD, van der Weijden T, von Meyenfeldt MF (2014) Sustainability of an enhanced recovery after surgery program (ERAS) in colonic surgery. World J Surg

10. Roulin D, Blanc C, Muradbegovic M, Hahnloser D, Demartines N, Hubner M (2014) Enhanced recovery pathway for urgent colectomy. World J Surg 38(8):2153-2159

11. Maessen J, Dejong CH, Hausel J, Nygren J, Lassen K, Andersen J, Kessels AG, Revhaug A, Kehlet H, Ljungqvist O, Fearon KC, von Meyenfeldt MF (2007) A protocol is not enough to implement an enhanced recovery programme for colorectal resection. Br J Surg 94(2):224-231

12. Dindo D, Demartines N, Clavien PA (2004) Classification of surgical complications: a new proposal with evaluation in a cohort of 6336 patients and results of a survey. Ann Surg 240(2):205-213

13. Pedziwiatr M, Kisialeuski M, Wierdak M, Stanek M, Natkaniec M, Matlok M, Major P, Malczak P, Budzynski A (2015) Early implementation of Enhanced Recovery After Surgery (ERAS®) protocol - compliance improves outcomes: a prospective cohort study. Int J Surg 21:75-81

14. Hendry PO, Hausel J, Nygren J, Lassen K, Dejong CH, Ljungqvist O, Fearon KC (2009) Determinants of outcome after colorectal resection within an enhanced recovery programme. Br J Surg 96(2):197-205

15. Alcantara-Moral M, Serra-Aracil X, Gil-Egea MJ, Frasson M, Flor-Lorente B, Garcia-Granero E (2014) Observational crosssectional study of compliance with the fast track protocol in elective surgery for colon cancer in Spain. Int $\mathrm{J}$ Color Dis 29(4):477-483

16. Bakker N, Cakir H, Doodeman HJ, Houdijk AP (2015) Eight years of experience with enhanced recovery after surgery in patients with colon cancer: impact of measures to improve adherence. Surgery 157 (6):1130-1136.

17. Gillissen F, Ament SM, Maessen JM, Dejong CH, Dirksen CD, van der Weijden T, von Meyenfeldt MF (2015) Sustainability of an enhanced recovery after surgery program (ERAS) in colonic surgery. World J Surg 39(2):526-533

18. Lyon A, Solomon MJ, Harrison JD (2014) A qualitative study assessing the barriers to implementation of enhanced recovery after surgery. World J Surg 38(6):1374-1380

19. Ljungqvist $O$ (2015) Sustainability after structured implementation of ERAS protocols. World J Surg 39(2):534-535

20. Cakir H, van Stijn MF, Lopes Cardozo AM, Langenhorst BL, Schreurs WH, van der Ploeg TJ, Bemelman WA, Houdijk AP (2013) Adherence to enhanced recovery after surgery and length of stay after colonic resection. Color Dis 15 (8):1019 1025.

21. Smart NJ, White P, Allison AS, Ockrim JB, Kennedy RH, Francis NK (2012) Deviation and failure of enhanced recovery after surgery following laparoscopic colorectal surgery: early prediction model. Color Dis 14(10):e727-e734 